\title{
A new Polyaniline nanocomposite with the photoadduct of hexaminecobalt(III) chloride metal complex and ethylenediamine ligand
}

\author{
Waseem Naqash* and Kowsar Majid ${ }^{\#}$ \\ Department of Chemistry, National institute of Technology, Srinagar, J\&K, 190006 \\ Email:*waseemnaqash@gmail.com,\#kowsarmajid@rediffmail.com
}

Organic-inorganic nanocomposites based on conducting polymers have attained many scientific and technological interests. The incorporation of nano sized inorganic filler into the polymer matrix produces a material with complimentary behavior between the polymer and inorganic counterpart [1]. These novel materials find applications in many industrial fields like nano scale electronics, electrochromic displays, light emitting diodes, sensors, electrochemical capacitors, corrosion inhibitors, photo-detectors, photocatalysts, etc. $[2,3]$. Therefore, in this direction the present work reports the synthesis of a new polyaniline (PANI) nanocomposite with the photoadduct of hexaaminecobalt(III) chloride $\left(\left[\mathrm{Co}\left(\mathrm{NH}_{3}\right)_{6}\right] \mathrm{Cl}_{3}\right)$ metal complex and ethylenediamine ligand (en) as filler via in situ oxidative polymerization by ammonium persulphate in non-aqueous DMSO medium. The dopant metal complex has been synthesized by known literature method. The photoadduct of the complex has been synthesized by irradiating its aqueous solution in presence of en ligand. The photo-substitution of ligands and hence successful synthesis of photoadduct has been proved by recording change in $\mathrm{pH}$ and taking $\mathrm{UV}-\mathrm{Vis}$ spectra before and after irradiation. The as synthesized photoadduct was reduced in size by high energy ball milling and subsequently used as filler in PANI matrix during its in situ polymerization to form PANI/photoadduct nanocomposite. The synthesized photoadduct and nanocomposite was characterized by UV-Vis, FTIR, XRD, and SEM analysis. UV-Vis and FTIR spectra prove successful interaction between the PANI and photoadduct microcrystals. XRD spectra show crystalline and semi-crystalline nature of photoadduct and nanocomposite respectively. Thermal analysis reveals increase in the thermal stability of nanocomposite as compared to that of pure PANI. The electrical study shows less conducting nature of PANI/photoadduct nanocomposite as compared to that of PANI as shown in Figure 1.
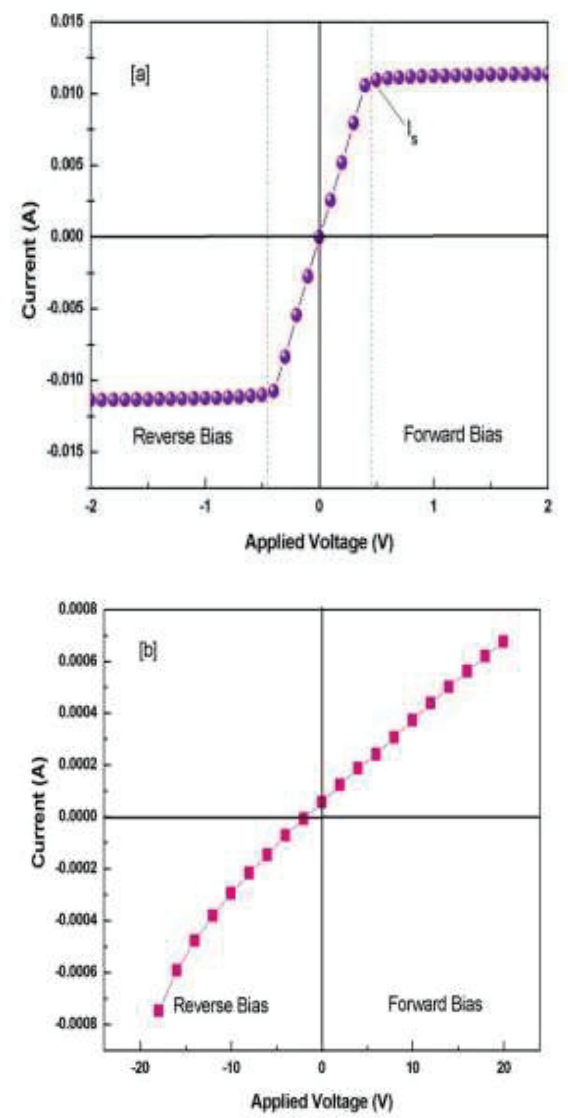

Figure 1: I-V curves of (a) PANI and (b) nanocomposite

\section{References}

1. M. Khairy, M.E. Gouda. J. Adv. Res. 6 (2015) $555-562$

2. D.E. Stilwell, S.M. Park, J. Electrochem. Soc. 135 (1988) 2491

3. M.A. Rahman, P. Kumar, D. Park, Y. Shim, Sensors 8 (2008) 118 